\title{
A Novel Role for Oligodendrocyte Precursor Cells (OPCs) and Sox 10 in Mediating Cellular and Behavioral Responses to Heroin
}

\author{
Jennifer A Martin 1,3, Aaron Caccamise ',3, Craig T Werner', Rathipriya Viswanathan', Jessie J Polanco', \\ Andrew F Stewart', Shruthi A Thomas', Fraser J Sim' and David M Dietz ${ }^{*, 1,2}$ \\ 'Program in Neuroscience, Department of Pharmacology and Toxicology, The State University of New York at Buffalo, Buffalo, NY, USA; \\ ${ }^{2}$ Department of Psychology, Research Institute on Addictions, The State University of New York at Buffalo, Buffalo, NY, USA
}

\begin{abstract}
Opiate abuse and addiction have become a worldwide epidemic with great societal and financial burdens, highlighting a critical need to understand the neurobiology of opiate addiction. Although several studies have focused on drug-dependent changes in neurons, the role of glia in opiate addiction remains largely unstudied. RNA sequencing pathway analysis from the prefrontal cortex (PFC) of male rats revealed changes in several genes associated with oligodendrocyte differentiation and maturation following heroin self-administration. Among these genes changed was Sox 10, which is regulated, in part, by the chromatin remodeler BRGI/SMARCA4. To directly test the functional role of SoxIO in mediating heroin-induced behavioral plasticity, we selectively overexpressed SoxIO and BRGI in the PFC. Overexpression of either Sox 10 or BRGI decreased the motivation to obtain heroin infusions in a progressive ratio test without altering the acquisition or maintenance of heroin self-administration. These data demonstrate a critical, and perhaps compensatory, role of Sox 10 and BRGI in oligodendrocytes in regulating the motivation for heroin.
\end{abstract}

Neuropsychopharmacology (2018) 43, I385-1394; doi: I0.1038/npp.20 I7.303; published online 24 January 2018

\section{INTRODUCTION}

Drug addiction is a large societal and financial burden on the United States, with more than $\$ 190$ billion dollars spent annually on costs associated with addiction. Opiate addiction, including heroin use and abuse, has risen by more than $50 \%$ in the past decade (Dart et al, 2015), leading to a nationwide opiate epidemic. This is in part due to one of the hallmark characteristics of drug addiction, namely persistent and compulsive drug seeking that leads to relapse. Although studies have progressed our knowledge of how drug use can transition from acute to persistent drug taking, the underlying neuroadaptations that control the motivation to seek the drug remain understudied. A better understanding of the neurobiology underlying these behaviors is critically needed to aid the development of pharmacotherapies.

The uncontrollable drive to seek drugs and the diminished motivation to seek nondrug rewards are trademarks of substance abuse (Goldstein and Volkow, 2002; Goldstein and Volkow, 2011; Kalivas et al, 2005). These behaviors have

*Correspondence: Dr DM Dietz, Program in Neuroscience, Depart-
ment of Pharmacology and Toxicology, The State University of New
York at Buffalo, 3435 Main Street, 613 Biomedical Research Building,
Buffalo, NY I42 I4, USA, Tel: + I 716829 207I; Fax: + I 716829280 I;
E-mail: ddietz@buffalo.edu
3These authors contributed equally to this work.
Received 28 August 20 I7; revised 28 November 2017; accepted I I Received 28 August 2017; revised 28 November 2017; accepted II
December 2017; accepted article preview online 20 December 2017 been attributed to persistent alterations in the prefrontal cortex (PFC), a region directly connected to other key regions of the mesolimbic dopamine reward system (Koob and Volkow, 2010). The PFC is heavily involved in cognition, working memory, and executive function (Kennerley and Walton, 2011), all of which are critical components of drug addiction (Goldstein and Volkow, 2011). Previous studies have demonstrated critical roles for the PFC in drug craving (Capriles et al, 2003; Koya et al, 2006; McFarland et al, 2004; Schmidt et al, 2005), along with encoding reward-related memories (Kalivas et al, 2005), including those specifically related to heroin (Schmidt et al, 2005).

The studies that have begun to dissect the role of the PFC in opiate addiction have primarily focused on neurons, leaving the role of non-neuronal cells (eg, glia) in regulating drug-related behaviors largely understudied (for review, see Cadet and Bisagno, 2014 and Miguel-Hidalgo, 2009). Oligodendrocyte precursor cells (OPCs) are glial stem/ progenitor cells within the central nervous system (CNS) that, in response to the expression of various transcription factors, can proliferate and differentiate into oligodendrocytes. These oligodendrocytes support neuronal survival and are essential for efficient action potential propagation (Emery et al, 2009; Fields, 2005; Tognatta and Miller, 2016). Disruptions of OPC and oligodendrocyte gene expression have been implicated in psychiatric diseases such as major depression (Aston et al, 2005; Birey et al, 2015; Liu et al, 2012; Makinodan et al, 2012) and schizophrenia 
(Iwamoto et al, 2005). Although several studies have documented the involvement of oligodendrocytes in addiction, the focus has been on psychostimulants and ethanol (Arezoomandan et al, 2016; Mandyam et al, 2007; Navarro and Mandyam, 2015; Vargas et al, 2014; George et al, 2008). A more mechanistic and causal role for glia has yet to be explored in the context of heroin exposure.

The aim of this study was to investigate the role of OPC and oligodendrocyte gene expression in the PFC on the motivation to take opiates and drug-taking behaviors. Sox10 is a member of the Sry-related high-mobility group box (Sox) E family of transcription factors and is a lineage marker of oligodendroglia (for review, see Weider and Wegner, 2017) that is functionally important for regulating oligodendrocyte differentiation and myelin induction (Wang et al, 2014). The expression of Sox10 is regulated by the chromatin remodeler BRG1, which has an important role in drug-induced plasticity (Wang et al, 2016). Here we demonstrate that heroin self-administration induces Sox10 expression via BRG1 in the PFC and that its overexpression reduces the motivation to seek heroin.

\section{MATERIALS AND METHODS}

\section{Animals}

Male Sprague-Dawley rats weighing 250-300 g (Envigo Laboratories, Indianapolis, IN) at the beginning of the experiments were housed at $22-25^{\circ} \mathrm{C}$ under a $12: 12$ reverse light-dark cycle with lights on at $1800 \mathrm{~h}$ and ad libitum access to food and water. Testing took place $7 \mathrm{~d} /$ week in the dark portion of the light-dark cycle. All experiments were conducted in accordance with the Institutional Animal Care and Use Committee of The State University of New York at Buffalo.

\section{Drugs}

Solutions of heroin hydrochloride $(0.07 \mathrm{mg} / \mathrm{ml}$; generously gifted from the NIDA drug supply program) in $0.9 \%$ sterile saline were prepared weekly. The pump durations for infusions were adjusted according to body weight on a daily basis, to ensure the delivery of the correct dose of drug for each animal.

\section{Self-Administration Test Chambers}

The experimental chambers have been described elsewhere (Gancarz et al, 2015; Sun et al, 2016; Wang et al, 2016). Briefly, 24 standard Med Associates Inc. (St. Albans, VT) chambers containing two nose-poke holes each with infrared monitoring were used. Two stimulus lights were mounted above each nose-poke hole, with a house light in the center back wall of the test chamber. All chambers are housed in sound-attenuating boxes and controlled through a Med Associates interface.

\section{Jugular Catheterization and Patency}

The animals were implanted with chronic indwelling jugular catheters, as previously described (Gancarz et al, 2015; Wang et al, 2016). To preserve patency, the catheters were flushed daily with $0.2 \mathrm{ml}$ of heparinized saline $(50 \mathrm{IU} / \mathrm{ml})$ containing enrofloxacin $(4 \mathrm{mg} / \mathrm{ml})$. Catheter patency was tested once per week throughout the duration of the experiments by observing behavioral responses (loss of righting reflex and muscle tone) to an injection of ketamine hydrochloride $(5 \mathrm{mg} / \mathrm{ml}$ in $0.5 \mathrm{ml}$, i.v.). Only data from rats with patent catheters were included in analyses.

\section{Heroin Self-Administration}

The rats were trained to self-administer heroin $(0.02 \mathrm{mg} / \mathrm{kg} /$ inf) or saline for $3 \mathrm{~h}$ each day for a total of $10 \mathrm{~d}$ beginning 1 week after jugular catheter surgery. Responses in the active nose-poke holes resulted in an infusion of drug, followed by a $20 \mathrm{~s}$ time-out period using a fixed ratio (FR) 1 schedule of reinforcement; this was increased daily from the first day to the third to an FR3 schedule, which was maintained for the remainder of the experiment. Responses in the inactive hole had no programed consequences. Following each selfadministration session, the catheters were flushed and the rats were returned to the colony room. Twenty-four hours after the last self-administration session, the rats were rapidly decapitated and their brains were collected and sectioned into $1 \mathrm{~mm}$-thick sections. From the prefrontal cortical regions (according to Paxinos and Watson, 2005), $2 \mathrm{~mm}$ diameter biopsy punches were taken, ensuring that cortical tissue devoid of callosal fibers was reliably obtained.

\section{Viral Overexpression of Sox10 and BRG1 in the PFC}

Lentiviral vectors encoding either Sox10-HA or mCherry (lenti-Sox10 or lenti-mCherry, respectively) under the control of an EF1 $\alpha$ promoter were prepared as previously described (Wang et al, 2014). In addition, we also utilized a lentiviral vector encoding BRG1-Flag under the control of the Sox10:MCS5 promoter (Wang et al, 2014; VectorBuilder) for glial lineage expression (lenti-BRG1); all virus titers were $>1.0 \times 10^{7}$. The rats were assigned to receive prelimbic PFC injections of lenti-mCherry, lenti-Sox10, or lenti-BRG1; the injectors were set at a $10^{\circ}$ angle with coordinates of $\mathrm{AP},+3.2$; $\mathrm{ML},+1.2$; and DV, $-3.2 \mathrm{~mm}$ (Paxinos and Watson, 2005). The viruses were manually infused over $10 \mathrm{~min}(0.2 \mu \mathrm{l} / \mathrm{min}$, total infusion volume of $2 \mu \mathrm{l}$ ), and the needles were left in place for $10 \mathrm{~min}$ to allow for diffusion. Lenti-mCherry was injected to serve as the control for behavioral and cellular changes that are normally induced by heroin selfadministration. The animals were allowed 1 week to recover before jugular catheter implantation, which was conducted as previously described.

\section{Heroin Self-Administration and Progressive Ratio After Sox10/BRG1 Overexpression}

The animals were trained to self-administer heroin as described above, with the exception that the FR schedule was gradually increased from FR1 to FR10 over 13 days of training. After training, the animals were allowed to selfadminister heroin on a progressive ratio (PR) schedule of reinforcement for $6 \mathrm{~h}$. The session ended if no infusions were delivered within $1 \mathrm{~h}$ of the last infusion. The schedule of delivery increased after each infusion, using the progression $1,2,3,4,6,8,12,24,32$, 48, etc. (Gancarz et al, 2012). After 
testing, the catheters were flushed with heparin-saline and the animals were returned to the colony room.

\section{Cue-Induced Reinstatement After Sox10 Overexpression}

A separate group of animals were trained to self-administer heroin, as described above. Extinction tests were initiated $24 \mathrm{~h}$ after the last training session, as previously described (Wang et al, 2016). Briefly, the rats were placed in dark experimental chambers and their responses were recorded but resulted in no programmed consequence. Extinction sessions continued until responding fell to fewer than 20 responses per session. Twenty-four hours after the last extinction session, the animals were placed back in the chambers for a $1 \mathrm{~h}$ cue-induced reinstatement test, during which the active responses produced cues previously paired with drug delivery.

\section{RNA-Sequencing}

RNA libraries were prepared using the Illumina TruSeq RNA library preparation kit and sequenced on the Illumina HiSeq 2500 system. A 50-cycle single-end flow cell was used. Alignments were performed against the RN5 (rat) genome built using the Tophat (version 2.0.13) alignment algorithm and the UCSC refGene annotation set. Differential expression analysis was performed using Cuffdiff under default parameters.

\section{Western Blotting}

PFC tissue punches were homogenized in $25 \mathrm{mM}$ Tris ( $\mathrm{pH}$ 8.0) buffer with $0.25 \mathrm{M}$ sucrose. Samples of extracted protein $(30 \mu \mathrm{g})$ were loaded on $4-15 \%$ gradient Tris- SDS polyacrylamide gels for separation via electrophoresis and were then transferred to a nitrocellulose membrane and blocked in $5 \%$ nonfat milk or bovine serum albumin (BSA). All membranes were incubated overnight at $4^{\circ} \mathrm{C}$. The primary antibodies were diluted in blocking buffer (Rockland Immunochemicals, Inc., Limerick, PA), including rabbit anti-Sox10 (1:100; Santa Cruz Biotechnology, Dallas, TX), rabbit anti-BRG1 (1:2000; Abcam, Cambridge, MA), and mouse anti- $\beta$-actin ( 1 : 10000 ; Cell Signaling Technologies, Inc., Danvers, MA). The membranes were incubated for $1 \mathrm{~h}$ at room temperature with IRDye secondary antibodies ( $1: 5000$; LI-COR, Inc., Lincoln, NE). The membranes were imaged using an Odyssey infrared imaging system (LI-COR, Inc.) and quantified by densitometry using ImageJ (National Institutes of Health, Bethesda, MD) with normalization to the $\beta$-actin loading control.

\section{Immunostaining}

Twenty-four hours after the last self-administration session, rats were deeply anesthetized using Fatal Plus (Vortech Pharmaceuticals; $1 \mathrm{ml} / \mathrm{kg}$, i.p.) and then transcardially perfused first with a solution containing $1 \mathrm{U} / \mathrm{ml}$ heparin in sterile phosphate-buffered saline (PBS) followed by $4 \%$ formaldehyde. Whole brains were stored in a $15 \%$ sucrose solution for $24 \mathrm{~h}$ and then transferred to a $30 \%$ sucrose solution for $48 \mathrm{~h}$; after sucrose cryoprotection, the brains were stored at $-20{ }^{\circ} \mathrm{C}$ until dissection. Coronal sections encompassing the PFC $(+3.7$ to +2.5 from bregma based on Paxinos and Watson, 2005) were sliced on a cryostat at a thickness of $30 \mu \mathrm{m}$, and every third section was collected; tissue sections containing the PFC were stored in sterile PBS containing $0.01 \%$ sodium azide at $4{ }^{\circ} \mathrm{C}$ until further use. The sections were first incubated in a blocking solution containing 50\% normal donkey serum (Jackson ImmunoResearch), $5 \%$ BSA fraction V (Gibco), and $0.5 \%$ Triton X-100 for $1 \mathrm{~h}$ at room temperature and then incubated overnight at $4{ }^{\circ} \mathrm{C}$ in primary antibody (1:500 anti-rabbit oligodendrocyte transcription factor-2 (Olig 2; no. 9610) or 1:400 anti-rabbit HA (no. 05-904), EMD Millipore) diluted in a solution containing $25 \%$ normal donkey serum, $2.5 \%$ BSA fraction $\mathrm{V}$, and $0.5 \%$ Triton X-100. After washing in PBS with Tween 20, the sections were incubated in blocking buffer for $1 \mathrm{~h}$ at room temperature and then $1 \mathrm{~h}$ at room temperature in secondary antibody (goat anti-rabbit IgG 488 (no. A 11034) or goat anti-mouse $\operatorname{IgG}_{3} 594$ (no. A 21155), Invitrogen) in a solution containing $25 \%$ normal donkey serum, $2.5 \%$ BSA fraction $\mathrm{V}$, and $0.5 \%$ Triton $\mathrm{X}-100$. Sections were mounted with Vectashield (H-1200; Vector Labs, Burlingame, CA) and were imaged with a $\times 20$ objective on an LSM 510 Meta confocal microscope; images were taken and scored by an investigator blinded to the experimental conditions.

\section{Chromatin Immunoprecipitation-Quantitative PCR}

Chromatin Immunoprecipitation (ChIP) was performed for BRG1 as previously described (Gancarz et al, 2015; Wang et al, 2016) with minor modifications. Briefly, two PFC punches from two rats taken $24 \mathrm{~h}$ after the last selfadministration session were pooled for each sample $(n=1)$. These punches were immediately fixed in $1 \%$ formaldehyde for $12 \mathrm{~min}$ and then quenched in $2 \mathrm{M}$ glycine for $5 \mathrm{~min}$. Chromatin was sheared using a Biorupter 300 (Diagenode Diagnostics, Seraing, Belgium) at $4{ }^{\circ} \mathrm{C}$ at a high sonication intensity for $30 \mathrm{~s}$ on and $30 \mathrm{~s}$ off for $10 \mathrm{~min}$ followed by a $10 \mathrm{~min}$ rest, which was repeated a total of three times. Chromatin fragment sizes of 250-1000 bp were verified using agarose gel electrophoresis. Magnetic sheep anti-rabbit beads (Invitrogen of Thermo Fisher Scientific, Waltham, MA) were incubated with anti-BRG1 antibody rotating overnight at $4^{\circ}$ C. After washing, $70 \mu \mathrm{l}$ of the magnetic bead/antibody slurry was incubated with the sheared chromatin for $16 \mathrm{~h}$ at $4{ }^{\circ} \mathrm{C}$; $5 \%$ of the sheared chromatin was saved as an input control. Samples were washed with $\mathrm{LiCl}$ and Tris-EDTA buffer. Reverse cross-linking took place overnight at $65^{\circ} \mathrm{C}$ and proteins and RNA were removed using proteinase $\mathrm{K}$ (Invitrogen) and RNase (Roche, Basel, Switzerland), respectively. DNA was purified using a DNA purification kit (Qiagen, Hilden, Germany). Immunoglobulin G was used to control for nonspecific binding. Quantitative PCR (qPCR) was performed to identify the binding of BRG1 to proximal promoter regions of the Sox 10 gene and to NFKB (forward, 5'-GAGCAGAGAGGACCTTCCCT-3'; reverse, 5' -CCGTCC GCCCGTCGCCCGTC-3') and Smad3 (forward, 5'-GCTGT AAGACCACCACTCCT-3'; reverse, 5'-TGTCTCTCGTCT CTCCTGG-3'). Amplification reactions were run in triplicates with iQ SYBR green (Bio-Rad Laboratories, Inc., Hercules, CA), and each sample was normalized to an immunoglobulin G control. Fold changes were calculated relative to the saline control. 


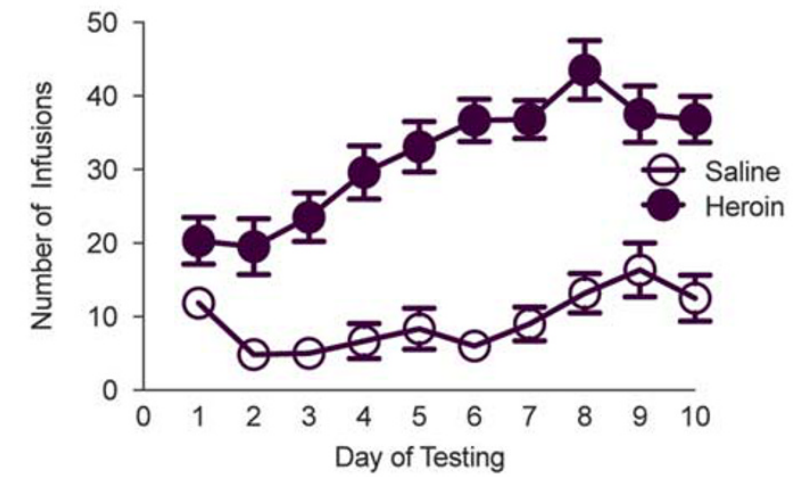

b

Pathway Analysis following heroin self-administration

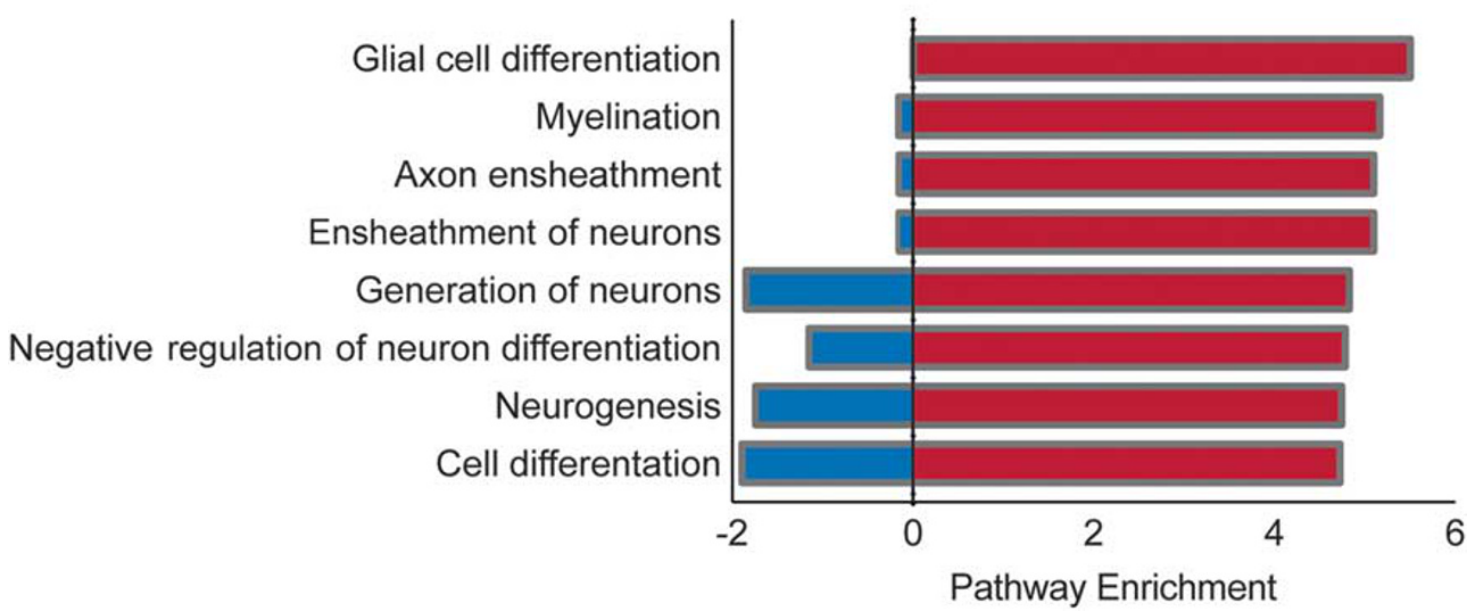

Figure I Heroin self-administration alters oligodendrocyte-associated gene expression. (a) Mean numbers of infusions of saline (open circles) or heroin (closed circles; $0.07 \mathrm{mg} / \mathrm{kg}$ per infusion, $3 \mathrm{~h} /$ day, $10 \mathrm{~d}$ ). (b) Graphical representation of RNA-sequencing (RNA-seq) pathway analysis following heroin selfadministration.

\section{Statistical Analyses}

All statistical analyses were performed using Prism (GraphPad Software, Inc., La Jolla, CA). Differences in selfadministration were analyzed by two-way repeated measures analyses of variance (ANOVAs). Differences in behavioral responding during the PR test were analyzed using a oneway ANOVA. The ANOVA was followed-up with an appropriate post-hoc test corrected for multiple comparisons when interactions were indicated. Differences in western blot and $\mathrm{qPCR}$ results and cell counts were analyzed using Student's $t$-tests. The criterion for statistical significance was a $P$-value of $<0.05$. All data are presented as means \pm SEMs.

\section{RESULTS}

\section{Upregulation of Myelin-Associated Genes Following Heroin Self-Administration}

Rats were trained $(3 \mathrm{~h} / \mathrm{d}$ for $10 \mathrm{~d})$ to nose poke for an intravenous infusion of saline or heroin (Figure 1a; two-way repeated-measures ANOVA, drug effect: $F_{1,140}=215.8$, $P<0.001$; session effect: $\mathrm{F}_{9,140}=4.988, P<0.001$; interaction effect: $\mathrm{F}_{9,140}=2.115, P=0.0320$ ). To analyze gene expression following heroin self-administration, RNA-sequencing (RNA-seq) was performed on PFC tissue punches taken $24 \mathrm{~h}$ after the last heroin exposure. A pathway analysis of RNA-seq results revealed an upregulation of glial cell differentiation and myelination pathways following heroin self-administration (Figure 1b; Supplementary Table S1) (Stolt et al, 2002), including a significant regulation of the key transcription factor Sox10 (saline vs. heroin, log fold change $(\log \mathrm{FC})=-0.826, P<0.01, n=3$ /group). In addition, a number of genes upregulated following heroin selfadministration, such as those encoding myelin basic protein (MBP) and myelin proteolipid protein, are under the control of Sox10 (Lee et al, 2008; Srinivasan et al, 2012), suggesting a key regulatory role for Sox10 in opiate-induced changes in glial pathways.

\section{Regulation of Sox10 in the PFC Following Heroin Self- Administration}

To examine whether the enrichment of Sox10 mRNA was leading to an increase in protein, we examined Sox10 protein 
a Heroin Self-Adminstration

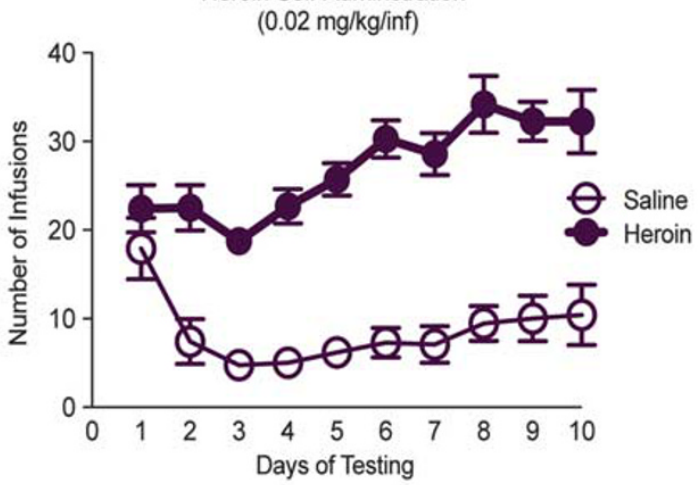

b

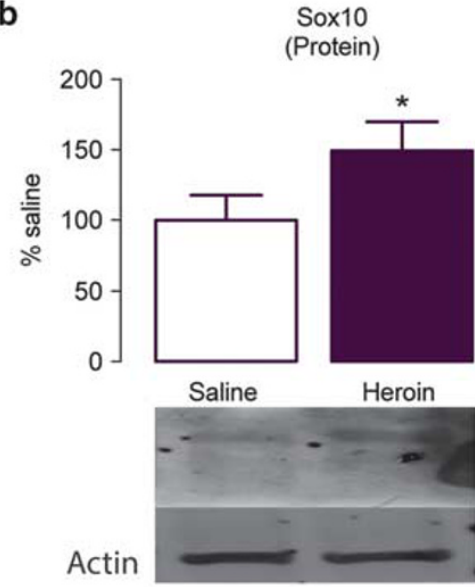

d

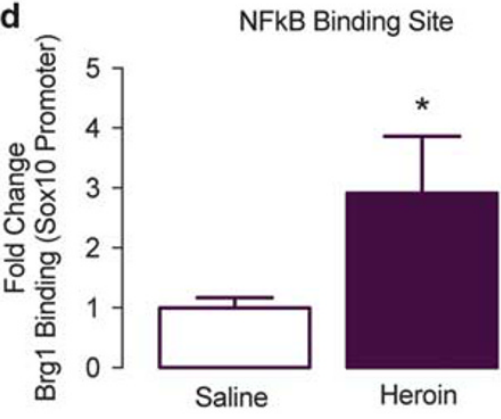

C
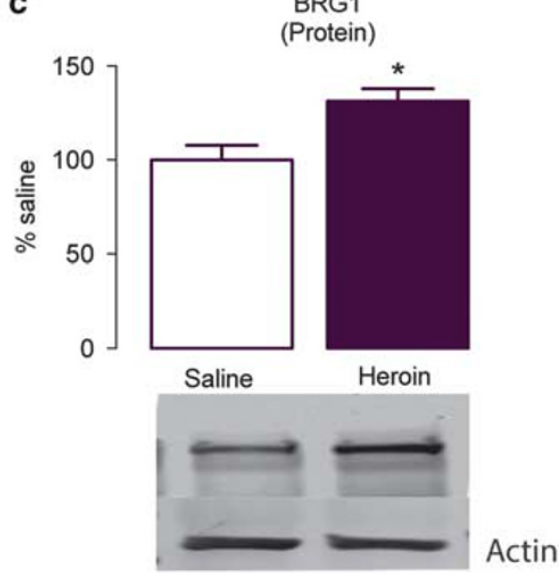

e

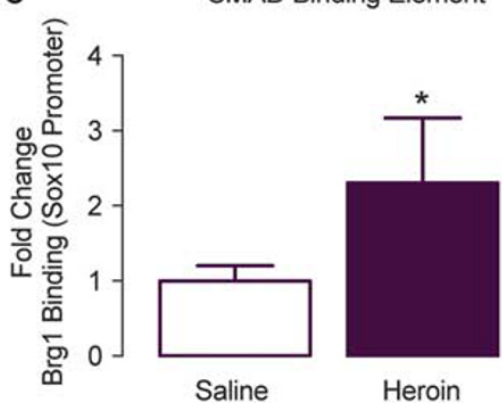

Figure $2 \mathrm{BRGI}$ in the prefrontal cortex (PFC) regulates Soxl0 expression following heroin self-administration. (a) Mean numbers of infusions of saline (open circles) or heroin (closed circles; $0.07 \mathrm{mg} / \mathrm{kg}$ per infusion, $3 \mathrm{~h} /$ day, $10 \mathrm{~d}$ ). Heroin self-administration resulted in an increase in Sox 10 (b) and BRG I (c) protein expression in the PFC. After heroin self-administration, there was an increase in BRGI occupancy at the NFkB (d) and Smad3 (e) binding elements of the Sox 10 promoter in the PFC as measured by quantitative chromatin immunoprecipitation (ChIP). Data are expressed as means \pm SEMs. $* P<0.05$.

via western blot analysis $24 \mathrm{~h}$ after the last heroin exposure (Figure 2a; two-way repeated-measures ANOVA, drug effect: $\mathrm{F}_{1,214}=296.1, \quad P<0.0001 ;$ session effect: $\mathrm{F}_{9,214}=4.313$, $P<0.001$; interaction effect: $\left.\mathrm{F}_{9,214}=3.17, P=0.0013\right)$. Sox10 protein expression was increased in the PFC compared with that from saline controls (Figure 2b; Student's $t$-test, $t_{9}=1.834, P=0.049 ; n=5-6 /$ group $)$, with no changes in the nucleus accumbens (NAc; Supplementary Fig. S1; Student's $t$-test, $t_{9}=0.639, P>0.05 ; n=5$-6/group). BRG1, a chromatin remodeler of the SWI/SNF complex, has been reported as a critical epigenetic mediator in the expression of Sox10 and in OPC differentiation and has been implicated in the epigenetic and transcriptional adaptions that occur after exposure to drugs of abuse (Bischof et al, 2015; He et al, 2016; Wang et al, 2016; Yu et al, 2013). Following heroin selfadministration, BRG1 protein expression in the PFC was increased compared with that from control saline animals (Figure 2c; Student's $t$-test, $t_{9}=2.989, P=0.015 ; n=5-6$ / group). To directly evaluate the transcriptional regulation of Sox10 mediated by BRG1 after heroin exposure, we performed a quantitative ChIP assay. Previous studies have demonstrated that BRG1 and NFKB form a transcriptional complex to regulate glial cell differentiation (Limpert et al, 2013); we sought to investigate if BRG1 was binding at NFKB sites to regulate Sox10 expression after exposure to heroin. We found there was an increase in BRG1 binding at NFKB 
binding elements on the Sox 10 promoter compared with that from saline controls (Figure 2d; Student's $t$-test, $t_{11}=2.509$, $P=0.014 ; n=5-8$ /group). In addition, given the known role of the BRG1-Smad3 complex in regulating responses to drugs of abuse (Wang et al, 2016), we next examined BRG1 binding at Smad3 DNA-binding sites and found that this interaction was indeed increased following heroin selfadministration (Figure 2e; Student's $t$-test, $t_{11}=1.832$, $P=0.047 ; n=5-8$ /group) compared with that from saline controls.

\section{Overexpression of Sox 10 in the PFC Decreases the Rewarding Effects of Heroin}

To examine the cellular consequences of manipulating Sox10 directly and via BRG1, we stereotaxically microinjected lentiviruses harboring transcripts for mCherry, Sox10, or Sox10-specific BRG1 (Figure 3a) into the prelimbic region of the PFC (Figure 3b). As expected, the Sox10-specific BRG1 lentivirus increased the expression of BRG1 protein (Figure $3 c$; Student's $t$-test, $t_{10}=1.846, P=0.047 ; n=5-7$ / group) as well as Sox10 (Figure 3d; Student's $t$-test, $t_{10}=2.23$,
$P=0.049 ; n=5-7 /$ group) compared with that from lentimCherry controls. Furthermore, compared with that from lenti-mCherry controls, intra-PFC injections of lenti-Sox10 increased the density of cells positive for Olig2, a transcription factor marker for the oligodendrocyte lineage (Weider et al, 2015) (Figure 3e; Student's $t$-test, $t_{6}=1.955, P=0.049$; $n=5-6 /$ group). Behaviorally, the overexpression Sox10 or BRG1 (via lenti-Sox10 or lenti-BRG1, respectively) did not result in any differences in the acquisition of heroin taking as measured by the number of infusions per test session across days of training (Figure 4a; two-way repeated-measures ANOVA, all $P$-values $<0.05, n=7-8$ /group). We next sought to determine whether the viral-mediated changes in BRG1 and Sox10 expression could alter the motivation to take heroin. To this end, animals were tested in a PR paradigm, which is thought be a model of motivation for obtaining a reinforcer (Pelloux et al, 2007). There was a main effect of viral manipulation on the number of active responses during the PR paradigm (Figure $4 \mathrm{~b} ; \mathrm{F}_{2,17}=16.19$, $P=0.0001, n=7-8$ /group). A post-hoc analysis determined that the overexpression of Sox10 or BRG1 in the prelimbic PFC resulted in fewer responses in the active hole $(P<0.01$
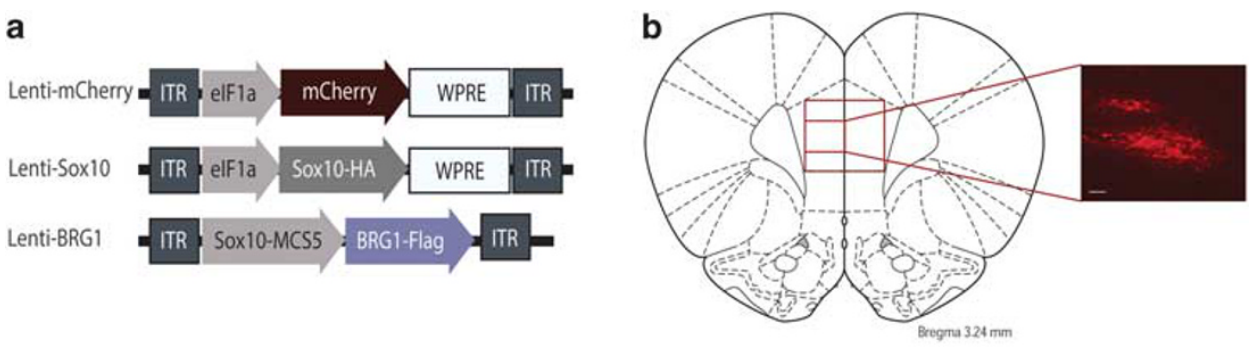

C

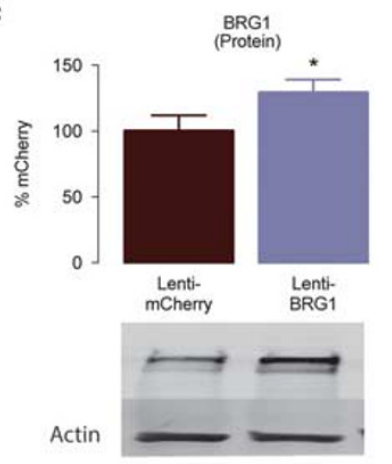

e

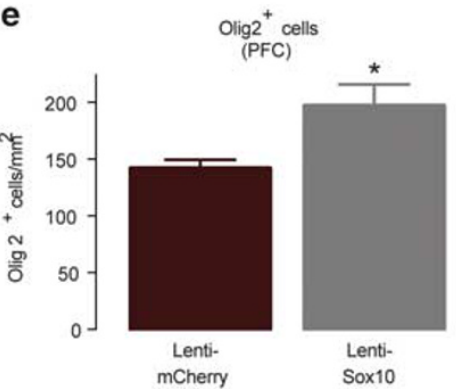

d
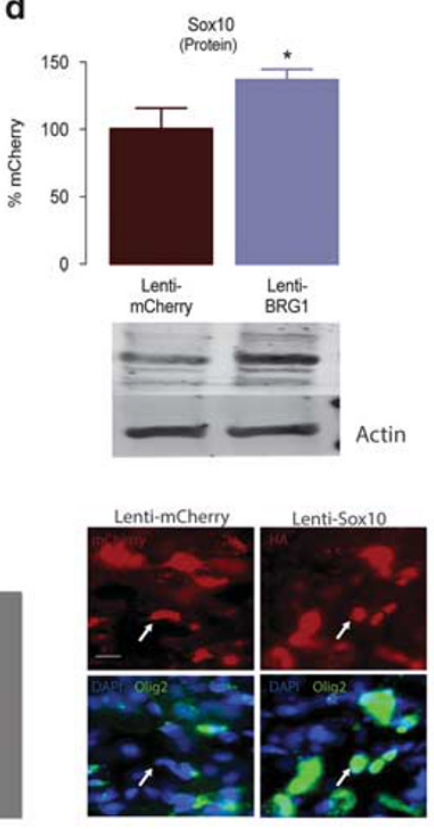

Figure $3 \mathrm{BRGI}$ and Sox 10 overexpression alter cellular plasticity following heroin self-administration. (a) Schematic of lenti-mCherry, lenti-Sox I0, and lentiBRGI virus constructs. (b) Representative image of a coronal section of the rat brain $(3.24 \mathrm{~mm}$ from bregma) depicting the virally-infected cells in the prefrontal cortex (PFC); scale bar, $25 \mu \mathrm{m}$. Lenti-BRGI injection led to increased protein expression of BRG I (c) and SoxI0 (d), whereas lenti-Sox I 0 increased the density of Olig2 ${ }^{+}$positive cells in the PFC; scale bar, $20 \mu \mathrm{m}$ used for all $I \mathrm{HC}$ images in panel (e). Data are expressed as means \pm SEMs. $* P<0.05$. 
a

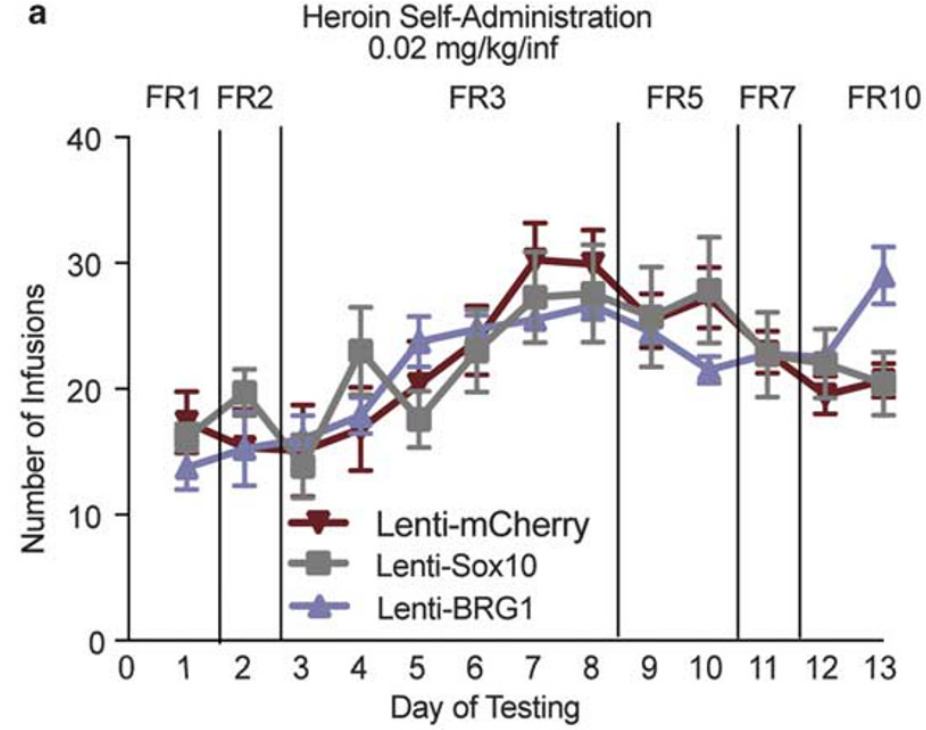

b

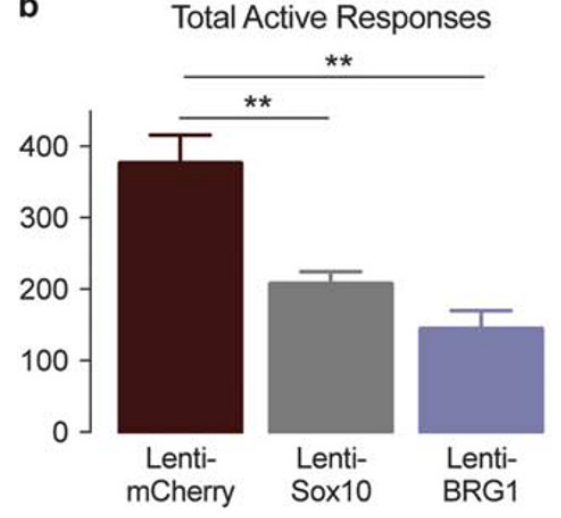

C

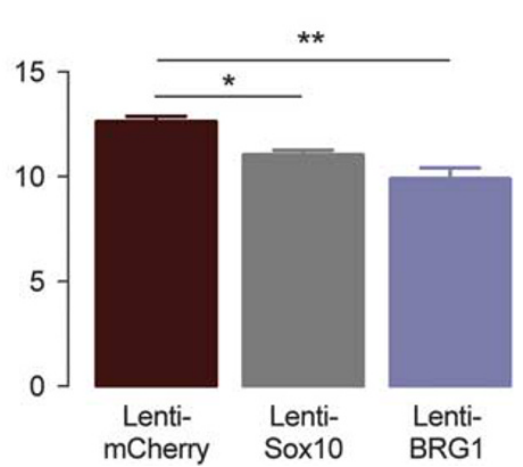

Figure 4 BRGI and SoxI0 overexpression specifically decrease the motivation to take heroin. (a) Overexpression of SoxI0 or BRGI (via lenti-SoxI0 or lenti-BRGI, respectively) did not alter the mean numbers of infusions self-administered per session ( $0.07 \mathrm{mg} / \mathrm{kg}$ per infusion). Overexpression of Sox 0 or $\mathrm{BRG}$ I resulted in decreased numbers of total active responses (b) and infusions earned (c) during the $6 \mathrm{~h}$ progressive ratio (PR) test. Data are expressed as means \pm SEMs. $* P<0.05$, $* * P<0.01$.

for both lenti-Sox10 and lenti-BRG1). Furthermore, this reduction in active responding resulted in significantly fewer infusions of heroin (Figure 4c; main effect of virus: $\mathrm{F}_{2,17}=11.89, P=0.0006, n=7-8$ /group), which was different in the lenti-Sox10 $(P<0.05)$ and lenti-BRG1 $(P<0.01)$ animals compared with that in lenti-mCherry controls, and a lower breakpoint (Supplementary Fig. S2; main effect of virus: $\left.\mathrm{F}_{2,17}=12.47, P=0.0005\right)$, which also differed in lentiSox10 and lenti-BRG1 animals compared with that from the lenti-mCherry controls (both $P<0.01$ ). Importantly, there were no differences in inactive responses (all $P>0.05$ ). In a separate group of animals, we found no changes in the responding of lenti-Sox10 animals during a cue-induced reinstatement test compared to that from lenti-mCherry controls (Supplementary Fig. S3; Student's $t$-test, $t_{13}=1.846$, $P=0.730 ; n=7-8$ /group).

\section{DISCUSSION}

Drug-induced neuronal cellular plasticity within the mesolimbic dopamine circuit is a well-established pathogenesis of the addicted phenotype (for review, see Lüscher, 2013 and Russo et al, 2010). Here we document a role for the lessstudied glial cells in regulating addiction-like behavior after exposure to heroin.

After heroin self-administration, there is an upregulation in transcripts from cellular pathways associated with glial cell differentiation and myelination in the PFC. Oligodendrocytes provide the myelin sheaths in the CNS that reduce the ATP energy requirement for restoring ion gradients in axonal fibers, while enhancing the speed of propagating signals (Nave, 2010). Some studies have begun to dissect the role of glial cells in addiction (for review see Miguel-Hidalgo, 2009), documenting a decrease in myelination within discrete regions of the mesolimbic dopamine system with exposure to cocaine and alcohol (Albertson et al, 2004; Kovalevich et al, 2012; Lewohl et al, 2000). In addition, Mandyam et al (2007) found that both short- and longaccess methamphetamine self-administration reduced the number of OPCs in the medial PFC positive for the neural/ glial antigen 2 marker, which would suggest there are fewer 
cells available to differentiate into mature myelinating oligodendrocytes.

Initial in vitro studies on the effects of opiates on oligodendroglial cells found that not only do oligodendroglial cells express the opioid receptors, but opiates have differential effects on cellular processes, including mitogenesis, DNA synthesis, and chromatin remodeling (Hauser et al, 1993; Knapp et al, 1998; Oguri et al, 1976). In vivo studies have shown an increase in MBP expression and other markers of mature oligodendrocytes in the NAc of pups from buprenorphine-treated mothers (Eschenroeder et al, 2012; Sanchez et al, 2008). Similarly, Vassoler et al (2017) identified a dysregulation of $\mathrm{MBP}$ in this region in the first- and second-generation offspring from a mother exposed to morphine during adolescence. To our knowledge, our study is the first to investigate the effects of opiates on adult OPCs in the PFC, and the adaptations we observed represent an understudied form of adult CNS plasticity that mediates behavioral outcomes such as drug taking.

Whereas psychostimulants such as methamphetamine are known to decrease myelin gene expression in the PFC (Mandyam et al, 2007), we found that the exposure to opiates increases the expression of oligodendrocyte lineage genes. It is interesting that both drug classes produce addiction-like behaviors in rodents, but the underlying neurobiological mechanisms mediating these behaviors appear to differ (Badiani et al, 2011).

Heroin self-administration leads to an increase in the expression of the SWI/SNF chromatin remodeler BRG1, which was previously shown to have an important role in regulating the expression of Sox10 (Stolt et al, 2002) and myelin-associated genes (Bischof et al, 2015; Limpert et al, 2013; Marathe et al, 2013). Consistent with this, we demonstrate that BRG1 binding at the promoter of Sox10 is increased at both the NFKB and Smad3 DNA-binding sites, suggesting that these transcriptional complexes may be critical for regulating the cellular plasticity induced by drugs of abuse (Gancarz et al, 2015, Wang et al, 2016). The BRG1mediated increase in Sox10 expression in the PFC may represent a neuroprotective mechanism to compensate for heroin-induced changes in white matter ( $\mathrm{Li}$ et al, 2013), as we do not see this increase in other areas of the mesolimbic dopamine system with low amounts of white matter, such as the NAc. Sox10 overexpression might potentiate the survival and integration of new oligodendrocytes, surpassing that induced by heroin, resulting in a greater abundance of Olig $2^{+}$ cells in animals injected with lenti-Sox10 than in lentimCherry controls.

The observed increases in Sox10 expression and the number of oligodendroglial lineage cells represent cellular plasticity within the PFC induced by heroin. This plasticity is observed as a change in the behavioral responses that characterizes the self-administration model of addiction. Surprisingly, overexpression of Sox10 did not exacerbate drug taking but rather attenuated the motivation to obtain the drug. This suggests that glial plasticity may occur as an attempt to compensate for heroin-induced changes. The medial portion of the PFC is known to have a direct role in modulating inhibitory control, which is disrupted in drug addiction and many other psychiatric disorders (Volkow et al, 2003). The prelimbic area has a critical role in encoding the value of rewards (Baldo, 2016) and is heavily involved in

action-outcome associations (Gourley et al, 2010), such as during the PR test in which the value of the reward (drug) diminishes as the effort to obtain it increases. We found that an increase in Sox10 either by viral overexpression directly or via lenti-BRG1 reduced the numbers of active responses and infusions earned, as well as the breakpoint, which indicates a reduction in motivation. These overexpression effects were specific for motivation, as we did not see any changes in a cue-induced reinstatement paradigm, an animal model of drug craving. Previous studies have shown discrepancies in responding between $\mathrm{PR}$ and FR schedules (O'Connor et al, 2010; Ramirez-Nino et al, 2013), and this may be due in part to the brain regions regulating the behaviors involved. For example, we saw an increase in Sox10 expression in the PFC, which is heavily involved in regulating motivated behaviors (PR schedule of reinforcement, Koob and Volkow, 2010), whereas there was no change in the NAc, a region heavily implicated in regulating responses to drug-associated cues (FR schedule of reinforcement, Wang et al, 2016). Although previous studies have implicated the PFC in other aspects of addicted-like behaviors (ie, relapse and reinstatement; McFarland et al, 2004), the data presented here shows a specificity for Sox10 in regulating motivated behaviors in the PFC, highlight a possible divergence of OPC-mediate anatomical pathways regulating opiate-induced behaviors (Gourley and Taylor, 2016). These findings are of great importance, as addiction is epitomized by long-lasting maladaptive neuroadaptations that drive the motivation to seek more drug (Mandyam and Koob, 2012), and thus shed further insight into OPC mediated mechanism driving these behaviors.

Importantly, previous data form our lab demonstrated that BRG1 is regulated in the NAc after exposure to drugs of abuse (Wang et al, 2016). The regulation of Sox10 through BRG1 in combination with viral vector in which BRG1 expression is under control of the Sox10-enhancer promoter further supports a critical role for BRG1 in OPCs dependent plasticity following heroin. Although we cannot exclude the possibility that the effects on BRG1 after heroin exposure are from cell types other than OPCs, our work here demonstrates a critical role of BRG1 in oligodendroglial lineage cells in the PFC. The studies presented here, along with our previous work (Wang et al, 2016), highlight a cell type and brain regions specific role for BRG1 in mediated druginduced behavioral and cellular plasticity.

How the increases in Sox10 expression and the number of oligodendroglial lineage cells translate to the observed behavioral plasticity requires further investigation. Birey et al (2015) demonstrated that ablation of OPCs in the PFC induced a neuropsychiatric (depression-like) phenotype, suggesting their importance in maintaining proper neural function. Therefore, following heroin self-administration, it is possible that Sox10 is stabilizing the OPC pool in the PFC, which may be secreting neurotrophic factors known to influence the activity and survival of surrounding neuronal cells (Du and Dreyfus, 2002), possibly including GABAergic interneurons. In addition, it is possible that oligodendrocytes are providing metabolic support to inhibitory axons (Lee et al, 2012), thus modifying their connectivity to other limbic structures. These cells may also be incorporated in ongoing adult myelination in the PFC (Norton and Poduslo, 1973), which is important for neuronal function as demonstrated by 
Xiao et al (2016), who demonstrated oligodendrocytes facilitate, and are key in the learning of new behaviors. The emergence of a compensatory mechanism may represent a fine-tuning of the system, a process that can be seen with exposure to other drugs of abuse, such as cocaine, which leads to neuroadaptations that unmask changes in both behavioral responses and cellular plasticity (LaPlant et al, 2010; Renthal et al, 2007).

The findings reported here reveal a novel mechanism of adult cellular plasticity within the CNS after chronic heroin exposure. Taken together, this and other studies deepen our understanding of potential areas of study towards a future therapeutic intervention for addiction.

\section{FUNDING AND DISCLOSURE}

This work was supported by the National Institutes of Health (no. NIDA R01DA037257 to DMD, NIDA R01DA037257-S1 to JAM, NMSS RG 5505-A-2 to FJS, NCATS UL1TR001412 to JJP, and NIGMS R25GM09545902). The heroin used in these studies was generously gifted by the NIDA Drug Supply Program. The authors declare no conflict of interest.

\section{ACKNOWLEDGMENTS}

We thank Neneyo Mate-Kole for his assistance in cell counting and Moriah M. Cobb for her assistance in carrying out western blot experiments.

\section{REFERENCES}

Albertson DN, Pruetz B, Schmidt CJ, Kuhn DM, Kapatos G, Bannon MJ (2004). Gene expression profile of the nucleus accumbens of human cocaine abusers: evidence for dysregulation of myelin. J Neurochem 88: 1211-1219.

Arezoomandan R, Moradi M, Attarzadeh-Yazdi G, Tomaz C, Haghparast A (2016). Administration of activated glial condition medium in the nucleus accumbens extended extinction and intensified reinstatement of methamphetamine-induced conditioned place preference. Brain Res Bull 125: 106-116.

Aston C, Jiang L, Sokolov BP (2005). Transcriptional profiling reveals evidence for signaling and oligodendroglial abnormalities in the temporal cortex from patients with major depressive disorder. Mol Psychiatry 10: 309-322.

Badiani A, Belin D, Epstein D, Calu D, Shaham Y (2011). Opiate versus psychostimulant addiction: the differences do matter. Nat Rev Neurosci 12: 685-700.

Baldo BA (2016). Prefrontal cortical opioids and dysregulated motivation: a network hypothesis. Trends Neurosci 39: 366-377.

Birey F, Kloc M, Chavali M, Hussein I, Wilson M, Christoffel DJ et al (2015). Genetic and stress-induced loss of NG2 glia triggers emergence of depressive-like behaviors through reduced secretion of FGF2. Neuron 88: 941-956.

Bischof M, Weider M, Küspert M, Nave K-A, Wegner M (2015). Brg1-dependent chromatin remodelling is not essentially required during oligodendroglial differentiation. J Neurosci 35: 21-35.

Cadet JL, Bisagno V (2014). Glial-neuronal ensembles: partners in drug addiction-associated synaptic plasticity. Front Pharmacol 5: 204.

Capriles N, Rodaros D, Sorge RE, Stewart J (2003). A role for the prefrontal cortex in stress- and cocaine-induced reinstatement of cocaine seeking in rats. Psychopharmacology (Berl) 168: 66-74.

Dart RC, Severtson SG, Bucher-Bartelson B (2015). Trends in opioid analgesic abuse and mortality in the United States. $N$ Engl J Med 372: 1573-1574.
Du Y, Dreyfus CF (2002). Oligodendrocytes as providers of growth factors. J Neurosci 68: 647-654.

Emery B, Agalliu D, Cahoy JD, Watkins TA, Dugas JC, Mulinyawe $\mathrm{SB}$ et al (2009). Myelin gene regulatory factor is a critical transcriptional regulator required for CNS myelination. Cell 138: 172-185.

Eschenroeder AC, Vestal-Laborde AA, Sanchez ES, Robinson SE, SatoBigbee C (2012). Oligodendrocyte responses to buprenorphine uncover novel and opposing roles of $\mu$-opioid- and nociceptin/ orphanin FQ receptors in cell development: Implications for drug addiction treatment during pregnancy. Glia 60: 125-136.

Fields RD (2005). Myelination: an overlooked mechanism of synaptic plasticity? Neuroscientist 11: 528-531.

Gancarz AM, Kausch MA, Lloyd DR, Richards JB (2012). Betweensession progressive ratio performance in rats responding for cocaine and water reinforcers. Psychopharmacology (Berl) 222: 215-223.

Gancarz AM, Wang Z-J, Schroeder GL, Damez-Werno D, Braunscheidel KM, Mueller LE et al (2015). Activin receptor signaling regulates cocaine-primed behavioral and morphological plasticity. Nat Neurosci 18: 959-961.

George O, Mandyam CD, Wee S, Koob GF (2008). Extended access to cocaine self-administration produced long-lasting prefrontal cortex-dependent working memory imparments. Neuropsychopharmacology 33: 2464-2482.

Goldstein R, Volkow N (2002). Drug addiction and its underlying neurobiological basis: neuroimaging evidence for the involvement of the frontal cortex. AM J Psychiatry 159: 1642-1652.

Goldstein RZ, Volkow ND (2011). Dysfunction of the prefrontal cortex in addiction: neuroimaging findings and clinical implications. Nat Rev Neurosci 12: 652-669.

Gourley SL, Lee AS, Howell JL, Pittenger C, Taylor JR (2010). Dissociable regulation of instrumental action within mouse prefrontal cortex. Euro J Neurosci 32: 1726-1734.

Gourley SL, Taylor JR (2016). Going and stopping: dichotomies in behavioral control by the prefrontal cortex. Nat Neurosci 19: 656.

Hauser KF, Gurwell JA, Bhat NR (1993). Endogenous opioid systems and the growth of oligodendrocyte progenitors: paradoxical increases in oligodendrogenesis as an indirect mechanism of opioid action. Glia 9: 157-162.

He D, Marie C, Zhao C, Kim B, Wang J, Deng Y et al (2016). Chd7 cooperates with Sox10 and regulates the onset of CNS myelination and remyelination. Nat Neurosci 19: 678-689.

Iwamoto K, Bundo M, Yamada K, Takao H, Iwayama-Shigeno Y, Yoshikawa T et al (2005). DNA methylation status of SOX10 correlates with its downregulation and oligodendrocyte dysfunction in schizophrenia. J Neurosci 25: 5376-5381.

Kalivas PW, Volkow N, Seamans J (2005). Unmanageable motivation in addiction: a pathology in prefrontal-accumbens glutamate transmission. Neuron 45: 647-650.

Kennerley SW, Walton ME (2011). Decision making and reward in frontal cortex: complementary evidence from neurophysiological and neuropsychological studies. Behav Neurosci 125: 297-317.

Knapp P, Maderspach K, Hauser K (1998). Endogenous opioid system in developing normal and jimpy oligodendrocytes- $\mu$ and $\mathrm{k}$ opioid receptors mediate differential mitogenic and growth responses. Glia 22: 189-201.

Koob GF, Volkow ND (2010). Neurocircuitry of addiction. Neuropsychopharmacology 35: 217-238.

Kovalevich J, Corley G, Yen W, Rawls SM, Langford D (2012). Cocaine-induced loss of white matter proteins in the adult mouse nucleus accumbens is attenuated by administration of a $\beta$-lactam antibiotic during cocaine withdrawal. Am J Pathol 181: 1921-1927.

Koya E, Spijker S, Voorn P, Binnekade R, Schmidt ED, Schoffelmeer AN et al (2006). Enhanced cortical and accumbal molecular reactivity associated with conditioned heroin, but not sucroseseeking behaviour. J Neurochem 98: 905-915.

LaPlant Q, Vialou V, Covington HE 3rd, Dumitriu D, Feng J, Warren BL et al (2010). Dnmt3a regulates emotional behavior 
and spine plasticity in the nucleus accumbens. Nat Neurosci 13: 1137-1143.

Lee KE, Nam S, Cho E, Seong I, Limb JK, Lee S et al (2008). Identification of direct regulatory targets of the transcription factor Sox10 based on function and conservation. BMC Genomics 9: 408.

Lee Y, Morrison BM, Li Y, Lengacher S, Farah MH, Hoffman PN et al (2012). Oligodendroglia metabolically support axons and contribute to neurodegeneration. Nature 487: 443-448.

Lewohl JM, Wang L, Miles MF, Zhang L, Dodd PR, Harris RA (2000). Gene expression in human alcoholism: microarray analysis of frontal cortex. Alcohol Clin Exp Res 24: 1873-1882.

Li W, Li Q, Zhu J, Qin Y, Zheng Y, Chang H et al (2013). White matter impairment in chronic heroin dependence: a quantitative DTI study. Brain Res 1531: 58-64.

Limpert AS, Bai S, Narayan M, Wu J, Yoon SO, Carter BD et al (2013). NF-kappaB forms a complex with the chromatin remodeler BRG1 to regulate Schwann cell differentiation. J Neurosci 33: 2388-2397.

Liu J, Dietz K, DeLoyht JM, Pedre X, Kelkar D, Kaur J et al (2012). Impaired adult myelination in the prefrontal cortex of socially isolated mice. Nat Neurosci 15: 1621-1623.

Lüscher C (2013). Drug-evoked synaptic plasticity causing addictive behavior. J Neurosci 33: 17641-17646.

Makinodan M, Rosen KM, Ito S, Corfas G (2012). A critical period for social experience-dependent oligodendrocyte maturation and myelination. Science 337: 1357-1360.

Mandyam CD, Koob GF (2012). The addicted brain craves new neurons: putative role for adult-born progenitors in promoting recovery. Trends Neurosci 35: 250-260.

Mandyam CD, Wee S, Eisch AJ, Richardson HN, Koob GF (2007). Methamphetamine self-administration and voluntary exercise have opposing effects on medial prefrontal cortex gliogenesis. J Neurosci 27: 11442-11450.

Marathe HG, Mehta G, Zhang XL, Datar I, Mehrotra A, Yeung KC et al (2013). SWI/SNF enzymes promote SOX10-mediated activation of myelin gene expression. PLoS ONE 8: e69037.

McFarland K, Davidge SB, Lapish CC, Kalivas PW (2004). Limbic and motor circuitry underlying footshock-induced reinstatement of cocaine-seeking behavior. J Neurosci 24: 1551-1560.

Miguel-Hidalgo JJ (2009). The role of glial cells in drug abuse. Curr Drug Abuse Rev 2: 76-82.

Navarro AI, Mandyam CD (2015). Protracted abstinence from chronic ethanol exposure alters the structure of neurons and expression of oligodendrocytes and myelin in the medial prefrontal cortex. Neuroscience 293: 35-44.

Nave KA (2010). Myelination and support of axonal integrity by glia. Nature 468: 244-252.

Norton WT, Poduslo SE (1973). Myelination in rat brain: changes in myelin composition during brain maturation. J Neurochem $\mathbf{2 1}$ : 759-773.

O'Connor EC, Parker D, Rollema H, Mean AN (2010). The alpha4beta2 nicotinic acetylcholine-receptor partial agonist varenicline inhibits both nicotine self-administration following repeated dosing and reinstatement of niceotine seeking in rats. Psychopharmacology 208: 365-376.

Oguri K, Lee NM, Loh HH (1976). Apparent protein kinase activity in oligodendroglial chromatin after chronic morphine treatment. Biochem Pharmacol 25: 2371-2376.

Paxinos G, Watson C (2005). The Rat Brain in Stereotaxic Coordinates. Elsevier Academic Press.

Pelloux Y, Everitt BJ, Dickinson A (2007). Compulsive drug seeking by rats under punishment: effects of drug taking history. Psychopharmacology (Berl) 194: 127-137.

Ramirez-Nino AM, D'Souza MS, Markou A (2013). N-acetylcysteine decreases nioctine self-administration and cue-induced reinstatement of nicotine seeking in rats: comparison with the effects of $\mathrm{N}$-acetylcysteine on food responding and food seeking. Psychopharmacology 225: 472-482.

Renthal W, Maze I, Krishnan V, Covington HE 3rd, Xiao G, Kumar A et al (2007). Histone deacetylase 5 epigenetically controls behavioral adaptations to chronic emotional stimuli. Neuron 56: 517-529.

Russo SJ, Dietz DM, Dumitriu D, Morrison JH, Malenka RC, Nestler EJ (2010). The addicted synapse: mechanisms of synaptic and structural plasticity in nucleus accumbens. Trends Neurosci 33: $267-276$.

Sanchez ES, Bigbee JW, Fobbs W, Robinson SE, Sato-Bigbee C (2008). Opioid addiction and pregnancy: perinatal exposure to buprenorphine affects myelination in the developing brain. Glia 56: 1017-1027.

Schmidt ED, Voorn P, Binnekade R, Schoffelmeer AN, De Vries TJ (2005). Differential involvement of the prelimbic cortex and striatum in conditioned heroin and sucrose seeking following long-term extinction. Eur J Neurosci 22: 2347-2356.

Srinivasan R, Sun GN, Keles S, Jones EA, Jang SW, Krueger C et al (2012). Genome-wide analysis of EGR2/SOX10 binding in myelinating peripheral nerve. Nucleic Acids Res 40: 6449-6460.

Stolt CC, Rehberg S, Ader M, Lommes P, Riethmacher D, Schachner $M$ et al (2002). Terminal differentiation of myelinforming oligodendrocytes depends on the transcription factor Sox10. Genes Dev 16: 165-170.

Sun HS, Martin JA, Werner CT, Wang ZJ, Damez-Werno DM, Scobie KN et al (2016). BAZ1B in nucleus accumbens regulates reward-related behaviors in response to distinct emotional stimuli. J Neurosci 36: 3954-3961.

Tognatta R, Miller RH (2016). Contribution of the oligodendrocyte lineage to CNS repair and neurodegenerative pathologies. Neuropharmacology 110(Pt B): 539-547.

Vargas WM, Bengston L, Gilpin NW, Whitcomb BW, Richardson $\mathrm{HN}$ (2014). Alcohol binge drinking during adolescence or dependence during adulthood reduces prefrontal myelin in male rats. J Neurosci 34: 14777-14782.

Vassoler FM, Oliver DJ, Wyse C, Blau A, Shtutman M, Turner JR et al (2017). Transgenerational attenuation of opioid selfadministration as a consequence of adolescent morphine exposure. Neuropharmacology 113(Part A): 271-280.

Volkow ND, Fowler JS, Wang GJ (2003). The addicted human brain: insights from imaging studies. J Clin Invest 111: 1444-1451.

Wang J, Pol SU, Haberman AK, Wang C, O'Bara MA, Sim FJ (2014). Transcription factor induction of human oligodendrocyte progenitor fate and differentiation. Proc Natl Acad Sci USA 111: E2885-E2894.

Wang Z-J, Martin JA, Mueller LE, Caccamise A, Werner CT, Neve $\mathrm{RL}$ et al (2016). BRG1 in the nucleus accumbens regulates cocaine-seeking behavior. Biol Psychiatry 80: 652-660.

Weider M, Wegener A, Schmitt C, Kuspert M, Hillgartner S, Bosl MR et al (2015). Elevated in vivo levels of a single transcription factor directly convert satellite glia into oligodendrocyte-like cells. PLoS Genet 11: e1005008.

Weider M, Wegner M (2017). SoxE factors: transcriptional regulators of neural differentiation and nervous system development. Semin Cell Dev Biol 63: 35-42.

Xiao L, Ohayon D, McKenzie IA, Sinclair-Wilson A, Wright JL, Fudge AD et al (2016). Rapid production of new oligodendrocytes is required in the earliest stages of motor-skill learning. Nat Neurosci 19: 1210-1217.

Yu Y, Chen Y, Kim B, Wang H, Zhao C, He X et al (2013). Olig2 targets chromatin remodelers to enhancers to initiate oligodendrocyte differentiation. Cell 152: 248-261.

Supplementary Information accompanies the paper on the Neuropsychopharmacology website (http://www.nature.com/npp) 